\title{
Quantum optics experiment on the test of empty wave hypothesis
}

\author{
V.A. Skrebnev ${ }^{1}$ and M.V. Polski ${ }^{2}$ \\ ${ }^{1}$ Institute of Physics, Kazan Federal University, 18 Kremlyovskaya str., Kazan 420008, \\ Russia \\ email:vskrebnev@gmail.com \\ ${ }^{2}$ East-West University, 816 S. Michigan Ave., Chicago, \\ IL 60605, USA
}

\begin{abstract}
The experiment measured the absorption of single photons by absorbers with various absorption coefficients, in one of the beams, after the photons interacted with the beam splitter. The measurements showed that the absorption corresponds to single photon traveling in either one or another beam. The measurements support the original empty wave hypothesis which has been advanced in a number of works.
\end{abstract}

Key words: single photon experiment, subquantum processes, empty wave, de BroglieBohm theory

\section{Introduction}

In 1986 Grangier, Roger and Aspect [1] have demonstrated the interference of the two output beams from the beam splitter in experiments using single photon states, even though the photon can only be detected in one of the two output beams for a given run of the experiment. Paper [2] considers the hypothesis that one of the beams contains a wave which is not accompanied by a particle, i.e. an empty wave. A number of works suggest 
experiments for the detection of empty waves (see, e.g. [3-5]); however, until now no experimental evidence of the existence of such a wave has been received.

The Born rule connects the wave function to the probability density of finding the particle at a given point. However, this rule is not a basic law. In de Broglie-Bohm theory the wave is considered a physical reality, and the link between the probability density and the wave function has the status of a hypothesis. This means that the wave function may be not equal to zero even in the part of space where the particle is not observed. Hence, an empty wave may also be described by the wave function, which may explain the interference of the wave containing the photon, i.e. the probability wave, or full wave, and the empty wave. In paper [2] some assumptions are made which are true in the de Broglie-Bohm model.

Clearly in order to discuss the empty wave hypothesis it is important to understand the physical meaning of the wave function, i.e. to have an adequate interpretation of the wave function. We will not discuss various interpretations of wave function here. The fact that so many exist testifies to the absence of a satisfactory one. This is why many physicists lean towards the instrumentalist interpretation, best summed up in the succinct slogan "Shut up and calculate!" [6].

Like many physicists, we are uncomfortable with any of the commonly known interpretations. In our view, for a particle to be found in a point in space, it must actually be there at the moment when it is found. It is clear that for a particle to be manifested at a point in space, physical processes are needed to provide for this manifestation. In the early 1960ies, de Broglie formulated an approach adding a chance element to the movement of a particle; this chance element is caused by the particle's interaction with the hidden "subquantum environment". In papers $[7,8]$ we offer an interpretation of the wave function in which wave function is a consequence of processes taking place at the level of the organization of matter which underlies the phenomena described by quantum mechanics. We use the term "subquantum processes" for these processes. We think that 
the road into the structure of matter is a staircase with an infinite number of steps, and the subquantum level is one of these steps.

Experiments observe the manifestation of the particle in a certain point of the probability wave. It is common knowledge that behind every chance there is a rule. We do not know the rules of the subquantum world determining the formation of wave trains in which the particle manifests as an observable object. It is clear, however, that the photon can manifest anywhere in the wave train, and that this possibility is determined by some subquantum processes. A direct proof of the existence of an empty wave would mean that along with the wave train where the photon manifests, there also exists a wave train containing the potential possibility of the photon manifestation, but the actual photon does not manifest, and thus cannot be detected. Note that at any given time the photon manifests only in one of the points of the probability wave train. In all other points at that moment the wave may be considered empty.

According to [1], after the recombination of two beams an interference picture is observed. This implies that two coherent wave trains appear as a result of the interaction of the photon with the beam splitter. If the photon were to manifest in both wave trains, it would mean that its wave function is the superposition of two probability waves: the transmitted one and the reflected one. Note, that the photon can only be detected in one of the two output beams. The empty wave hypothesis is consistent with the suggestion that the photon manifests in only one of the two output beams. In this case the photon wave function cannot be a superposition of two probability waves. Thus, experiments are needed which will clarify whether the photon wave function after the interaction with the beam splitter is the superposition of two probability waves or the superposition of a probability wave and an empty wave.

\section{Experiment and discussion}

Our experiment is illustrated in figure 1. 


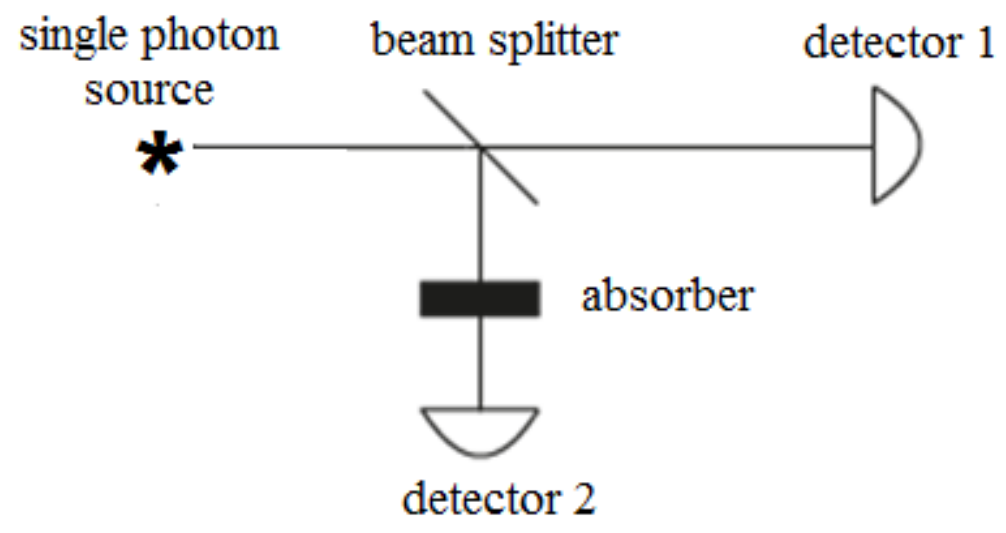

Fig.1

Let us consider that after the beam splitter the wave function of the single photon is the superposition of the wave functions corresponding to the transmitted and the reflected waves:

$$
\psi=c_{1} \psi_{t r}+c_{2} \psi_{r e}
$$

where the ratio of $\left|c_{1}\right|^{2}$ and $\left|c_{2}\right|^{2}$ is equal to the ratio of the numbers of photons registered by the detectors 1 and 2 in the absence of the absorber. Let $N_{0}$ is the number of the photons registered by both detectors in the absence of the absorber. Then numbers of the photons registered by the detector 1 and by the detector 2 will be equal to $N_{0}\left|c_{1}\right|^{2}$ and $N_{0}\left|c_{2}\right|^{2}$ accordingly.

If the number of the absorbed photons is $N_{a b}$, then the number of the unabsorbed photons is $\left(N_{0}-N_{a b}\right)$. Photon absorption has no effect on the wave function (1) of the unabsorbed photon after the beam splitter. If the wave function of the single photon were the superposition (1) and the absorber does not influence unabsorbed photons, then at the presence of the absorber the numbers of the photons registered by the detector 1 and by the detector 2 would be equal to $\left(N_{0}-N_{a b}\right)\left|c_{1}\right|^{2}$ and to $\left(N_{0}-N_{a b}\right)\left|c_{2}\right|^{2}$. The numbers of the photons registered by the detectors 1 and 2 would diminish on $N_{a b}\left|c_{1}\right|^{2}$ and $N_{a b}\left|c_{2}\right|^{2}$ accordingly. 
The measurement results are provided in Table 1.

\begin{tabular}{|l|l|l|l|l|l|}
\hline $\mathrm{k}$ & $\mathrm{T}$ & \multicolumn{1}{|c|}{$N_{1}$} & $N_{2}$ & $N_{1} / N_{2}$ & $\left(N_{1} / N_{2}\right)_{a v}$ \\
\hline 0 & 2 & 44730 & 33745 & 1.325 & \\
\hline 0 & 0.02 & 459 & 343 & 1.339 & 1.346 \\
\hline 0 & 0.002 & 45 & 33 & 1.373 & \\
\hline & & & & & \\
\hline 0.1 & 2 & 44271 & 26068 & 1.698 & \\
\hline 0.1 & 0.02 & 433 & 255 & 1.696 & 1.701 \\
\hline 0.1 & 0.002 & 43 & 25 & 1.710 & \\
\hline & & & & & \\
\hline 0.2 & 2 & 44188 & 20502 & 2.155 & \\
\hline 0.2 & 0.02 & 446 & 210.6 & 2.118 & 2.096 \\
\hline 0.2 & 0.002 & 44 & 28 & 2.016 & \\
\hline
\end{tabular}

Table 1. Column names:

$\mathrm{k}$ - absorption coefficient of the absorber

$\mathrm{T}=20 T_{m}$

$T_{m}$ - duration of one measurement cycle

$N_{1}$ - average number of photons registered by detector 1 in one measurement cycle $N_{2}$ - average number of photons registered by detector 2 in one measurement cycle $\left(N_{1} / N_{2}\right)_{a v}$ - average ratio of the number of photons registered by the detector 1 and the detector 2 at different $\mathrm{T}$ 
The table 1 shows that the ratio of the number of the photons registered by the detector 1 and the detector 2 without the absorber is 1.346 (with variation in the third decimal at various values of $\mathrm{T}$ ). This means, that in the absence of the absorber the number of the photons registered by the detector 1 is

$$
\left(N_{1}\right)_{0}=0.574 N_{0}
$$

and the number of the photons registered by the detector 2 is

$$
\left(N_{2}\right)_{0}=0.426 N_{0}
$$

Let us suppose that after the beam splitter the wave function of the single photon is the superposition

$$
\begin{gathered}
\psi=c_{1} \psi_{t r}+c_{2} \psi_{r e} \\
\left|c_{1}\right|^{2}+\left|c_{2}\right|^{2}=1
\end{gathered}
$$

where $\left|c_{1}\right|^{2}=0.574,\left|c_{2}\right|^{2}=0.426$.

Let us admit that the absorber does not influence unabsorbed photon and the photon wave function remains the superposition (4). Then, when absorber is present, the number of the photons registered by the detector 1 would be equal to $0.574\left(N_{0}-N_{a b}\right)$ and the number of the photons registered by the detector 2 would be equal to $0.426\left(N_{0}-N_{a b}\right)$. The ratio of the numbers of the photons registered by the detectors 1 and 2 would remain equal to the ratio 0.574 and 0.426 , i.e. equal to 1.346 . Our measurements have shown, however, that the presence of the absorber in the path of the reflected beam does not change the number of the photons registered by the detector 1 . In the presence of the absorber, the number of unabsorbed photons registered by the detector 2 corresponds up to the third decimal to the expression

$$
N_{2}=\left(N_{2}\right)_{0} 10^{-k}
$$

Because the presence of the absorber does not change the number of the photons registered by the detector 1 , the number of absorbed photons is equal to

$$
N_{a b}=\left(N_{2}\right)_{0}-N_{2}=\left(N_{2}\right)_{0}\left(1-10^{-k}\right)=0.426 N_{0}\left(1-10^{-k}\right)
$$


In the presence of the absorbers the ratio of the numbers of photons registered by the detectors 1 and 2 also up to the third decimal corresponds to the expression

$$
\frac{N_{1}}{\left(N_{2}\right)_{0} 10^{-k}}=1.346 * 10^{k}
$$

so it does change.

Let us suppose now that the photon wave function after the beam splitter is the superposition (4) and the absorber influences unabsorbed photon. Let us suppose also that as a result of the absorber influence the photon appears in the reflected beam (like an electron appears under influence of light in two slits experiments [9]). This photon will be registered by the detector 2 . Obviously, it may increase the number of the photons registered by the detector 2 and, accordingly, may decrease the number of the photons

registered by the detector 1 (but not to increase this number and to do it equal to $N_{0}\left|c_{1}\right|^{2}$ ). However, in our experiment formulas (2) and (5) describe the numbers of photons, registered by the detectors 1 and 2 without any amendments that could be a result of absorber influencing unabsorbed photons.

The results of our measurements, reflected in formulas (2), (5) and (7), correspond to the photon traveling after the beam splitter in either one or another beam. This means that the photon wave function after the interaction with the beam splitter is not the superposition of two probability waves. At the same time, for the interference in the experiments [1] to be observed, two waves must be superposed. This means that while the photon is traveling through one beam, the empty wave is traveling through the other beam. In other words, the wave function of the photon after the interaction with the beam splitter is the superposition of the probability wave (full wave) and the empty wave.

\section{Conclusion}

The results of our measurements and of experiment [1], combined together, demonstrate the existence of the empty wave. However, the need to confirm our results 
and to perform further experiments is obvious. For example, it would be very interesting to find out whether single-photon interference persists in the presence of an absorber.

Quantum mechanics is a fundamental theory which allows to describe a vast number of physical phenomena. However, as a truly fundamental theory, it cannot explain and describe itself. Accordingly, quantum mechanics says nothing about subquantum processes which provide for the existence of the empty wave.

The survey "Models of wave-function collapse, underlying theories, and experimental tests" [10] points out: "Quantum mechanics is an extremely successful theory... One should of course stay cautioned against assuming that quantum theory will be successful through and through ... The fact that a theory is extremely successful in one part of the parameter space should not be taken as a guarantee that it will continue to be successful in a different part of the parameter space ... And there are historical examples of longstanding successful theories eventually turning into approximations to more general theories when their extrapolation into a new part of the parameter space failed to be confirmed by experiment."

Experimental proof of the existence of the empty wave may be useful in developing a more general theory, to which quantum mechanics will be an approximation.

\section{References}

[1] P. Grangier, G. Roger and A. Aspect, Europhys. Lett. 1 (1986) 173.

[2] L. Hardy, "On the existence of empty waves in quantum theory", Physics Letters A, 167, issue 1, page 11 (1992).

[3] J. R. Croca, "An experiment for detection of empty waves", Physics Letters A, 124, no. 1,2 , page 22 , (1987)

[4] Peter E. Gordon, "Can empty waves be detected?", Physics Letters A, 138, no. 8, page $359,(1989)$

[5] Sofia Wechsler, About empty waves, their effect, and the quantum theory, 
arXiv:1008.4849v1 [quant-ph]

[6] Mermin, D.: Could Feynman Have Said This? Physics Today 57 (5) 10-11 (2004)

[7] V.A. Skrebnev, M.V. Polski, Some issues of quantum theory and subquantum processes, arXiv:1701.03019v4

[8] Polski, M.V., Skrebnev, V.A., An alternative derivation of canonical distribution as a result of irreversible processes in macrosystems, Eur. J. Phys. 38, 025101 (2017)

[9] The Feynman Lectures on Physics, Volume III, Chapter 1

[10] A. Bassi, et all, Models of wave-function collapse, underlying theories, and experimental tests, Reviews of Modern Physics, 85, 471 (2013). 